\title{
Comprensión de lectura desde la percepción de los docentes de inglés - lengua extranjera
}

\author{
Erika Yiseth Holguín Mariño ${ }^{1}$
}

Recibido: 26-07-2018

Aceptado: $21-09-2018$

\section{RESUMEN}

El presente artículo de investigación deriva de un interés personal por entender a detalle las creencias de algunos docentes de inglés como lengua extranjera de una universidad de carácter privado de la ciudad de Bucaramanga. El objetivo es entender cuales son las percepciones de algunos docentes de inglés- lengua extranjera acerca del rol que tiene la enseñanza de vocabulario en procesos de comprensión de lectura, dentro de un contexto universitario.

Para lograr el objetivo de la investigación se diseñó un estudio cualitativo de tipo fenomenológico cuya pretensión fue capturar la forma cómo los participantes percibieron el fenómeno, es decir el papel que desempeña la enseñanza de vocabulario en el proceso de comprensión de lectura. Se toma como población la participación de 4 docentes de una universidad privada y como insumos de análisis una entrevista semiestructurada diseñada para tal fin, y la guía cátedra, que es el documento con el que cuenta cualquier docente de inglés, adscrito al departamento de lenguas de la universidad. Para el análisis de la información se sigue el patrón propuesto por Creswell (2014) que sugiere una construcción lineal y jerárquica compuesta por varias etapas interrelacionadas.

1. Licenciada en Idiomas UIS- Aspirante a Magistra en Educación Universidad Autónoma de Bucaramanga.

Bucaramanga - Colombia.

Correo: erikaholguin@gmail.com Teléfono: 3045778626

ORCID: https://orcid.org/0000-0003-3962-303X

Google Académico: https://scholar.google.com/citations?hl=es\&user=Ju80 LwoAAAAJ

\&view_op=list_works\&gmla=AJsN-F55Pqe5V-\%20NfQ4Ruz9pT7mqowGqvD_47Wa_

a $1 \mathrm{hP} 2 \mathrm{HJvcWlg} 1$ B BxztydOOLXdsOyXTrON+3SvRgHx5vxllcxOqZT4llpa7A 
Como resultado se obtienen tres categorías: vocabulario como herramienta de comunicación, rol docente en la enseñanza de vocabulario, y relación entre lectura y vocabulario; cada una de las cuales contiene otras subcategorías de análisis donde se ubican los respectivos códigos de información producto de la entrevista realizada. Entre las estrategias resultantes para incrementar vocabulario resaltan: el uso de expresiones idiomáticas, la redefinición contextual, y la estrategia de recolección autónoma de vocabulario.

Palabras clave: centros educativos, liderazgo directivo, calidad educativa.

\title{
Reading comprehension from the perception of teachers of English - a foreign language
}

\begin{abstract}
The following research article derives from a personal interest on understanding meaningfully the believes of some English teachers from a private university in Bucaramanga city. The main objective is to understand which are the believes of some English teachers about the role vocabulary teaching have on reading comprehension process within a university context.

To reach the research goal a qualitative study was designed based on a phenomenological approach. Which pretention was to capture the way the phenomena was perceived by the participants. It means that vocabulary teaching role have on reading comprehension process. The population is composed by four professors from a private university and the data was taken from a semi structured interview (designed for that purpose) and the syllabi, which is the format that any English teacher, subscribed to the language department, has. To analyze the information, Creswell model (2014) was proposed and suggested a lineal and hierarchical construction composed by different and interrelated stages.
\end{abstract}

Three categories stood up: Vocabulary as communication tool, teacher's role on vocabulary teaching, and relationship between reading and vocabulary. Each category implies other analysis subcategories where 
the data codes produced from the interview process are located. From the resulting strategies to increase vocabulary we can have: use of idioms and slangs, contextual redefinition and the autonomous self-collection strategy.

Keywords: communicational interaction, vocabulary, learning, comprehension, perceptions, techniques, guide syllabi.

\section{Compréhension écrite de la perception des professeurs d'anglais - une langue étrangère}

\section{RÉSUMÉ}

Cet article d'investigation part d'un intérêt personnel afin de comprendre en détaille les croyances de certains professeurs d'anglais comme une langue étrangère dans une université prive à Bucaramanga. L'objectif est de comprendre quels sont les perceptions de certains professeurs $d$ 'anglais comme une langue étrangère dans une contexte universitaire.

Afin de réussir l'objectif de cette investigation on a désigné une étude qualitative de type phénoménologique qui veut savoir commet les participants perçoivent le phénome. Cet a dire le rôle que l'enseignement du vocabulaire a dans un procès de la compression de lecture. On prend comme population quatre enseignants d'une université privée et comme l'outil d'analyses un entretien semi structuré désigné pour cette investigation et la guide de chaire que c'est le document que toutes les professeures qui fassent part du département de langues de l'Université. Pour l'analyses de l'information, on suit le patron proposé par Creswell (2014) qui suggère une construction linéaire et hiérarchique compose par plusieurs d'étapes liées.

Comme résultat on obtient trois catégories : le vocabulaire comme l'outil de communication, le rôle du professeur en l'enseignement du vocabulaire et la relation entre la lecture et le vocabulaire. Chaque a ses sous-catégories d'analyse où on trouve les codes d'information comme résultat de l'entretien fait. Entre ces stratégies résultantes pour l'incrémentation du vocabulaire ressort : I'usage d'expressions 
idiomatiques, la redéfinition contextuelle et la stratégie de trouver des nouveaux mots de forme autonome.

Des mots clés: L'interaction communicative, le vocabulaire, l'apprentissage, la compréhension, la perception, les techniques, la guide chaire.

\section{Introducción}

En el año 2004 el Ministerio de Educación Nacional (MEN) dio inicio al Programa Nacional de Bilingüismo en su compromiso por mejorar las competencias comunicativas en lengua extranjera, desde ese momento, adquirió la obligación de revisar los fundamentos teóricos y filosóficos que apoyan los componentes del currículo para el aprendizaje y la enseñanza de lenguas en el país. Uno de los componentes básicos para el proceso de enseñanza- aprendizaje de una lengua extranjera incluye la pedagogía y las diferentes formas de instrucción y aprendizaje de vocabulario, componente ineludible en la interacción comunicativa, tanto de usuarios de la lengua materna, como de lengua extranjera (de Almeida, Santos y Porto, 2016).

A menudo, las instituciones educativas obvian la inclusión del componente de vocabulario en los planes de estudio, así como las estrategias que podrían ser de gran utilidad para los docentes que están encargados de este proceso de enseñanza en diferentes niveles de competencia (Azevedo, 2014). Esto combinado con estudios de cómo se deben enfocar los procesos de lectura, y el aprendizaje y enseñanza de vocabulario en lengua extranjera (Aebersold \& Field, 1997; Peregoy \& Boyle, 2013), ha motivado que los procesos de enseñanza y aprendizaje de vocabularios en lengua extranjera, y aun materna sean, seriamente, tenidos en cuenta como un componente vital del currículo.

En un esfuerzo por entender y para mejorar la enseñanza del vocabulario en lengua inglesa, se propuso este estudio, cuyo objetivo principal es identificar las percepciones de los docentes de inglés acerca del rol que tiene la enseñanza de vocabulario en el proceso de comprensión de lectura en estudiantes de pregrado. 
Se plantean además los siguientes objetivos específicos:

1. Describir las técnicas que utilizan los docentes para enseñar vocabulario en inglés como Lengua extranjera.

2. Caracterizar las percepciones de algunos docentes sobre la enseñanza de vocabulario.

3. Analizar el documento, guía cátedra, con el que cuenta un docente de inglés-lengua extranjera encargado de la enseñanza de inglés-lengua extranjera en estudiantes de pregrado

El objetivo principal corresponde al problema de investigación centrado en que son muchos los factores que interfieren en el proceso de comprensión de lectura de inglés como lengua extranjera, tanto a nivel de docentes como de aprendices (Aparicio \& Ostos, 2018). Sin embargo, la meta está enfocada al análisis de las percepciones de los docentes; quienes día a día deben enfrentar nuevos retos con aprendices de inglés a nivel de pregrado y que necesitan entender cómo hacer de sus prácticas educativas mejores experiencias de vida.

\section{Teorias vinculadas al proceso de aprendizaje}

El aprendizaje es una constante en el transcurso de nuestras vidas, continuamente estamos interactuando con otros y modificando conductas, por lo que resulta de vital importancia profundizar sobre los modelos y/o estrategias que se utilizan en el proceso de enseñanza y que de una u otra manera favorecen el aprendizaje en nuestros estudiantes (Viejo, cabezas y Martínez, 2013). Para Muñoz y Periáñez (2013) el aprendizaje se precisa como la modificación permanente de nuestra conducta, que se logra a través de las experiencias del individuo en su mundo real. Así, son múltiples las variaciones de conducta que pueden desarrollarse en el ser humano y que conllevan a un proceso de aprendizaje.

Diferentes enfoques y metodologías se hacen presentes en el quehacer de los maestros sobre las concepciones del proceso enseñanzaaprendizaje. Al respecto, se presenta un panorama general de las teorías constructivistas (Aparicio, 2018), entre ellas, El aprendizaje investigativo que enfatiza el crecimiento individual, la importancia del ambiente, y 
el rol del maestro en el proceso de enseñanza- aprendizaje, con el fin de desarrollar las habilidades cognitivas de los estudiantes. (Tracey \& Morrow, 2012). La teoría de los esquemas; que de acuerdo con Peredo (2005) es una de las más usadas en procesos de comprensión de lectura, ya que se tienen en cuenta las estructuras previas de los aprendices para abordar un nuevo conocimiento. Por su parte, Rosenblatt (1996) presenta la teoría transaccional de la lectura, según la cual toda experiencia de lectura es única a cada individuo y se convierte en una experiencia transaccional compleja en la medida que involucra cada ser humano con sus propios esquemas de conocimiento, sus experiencias afectivas y vivenciales, y el contexto sociocultural donde se lleva a cabo el proceso.

La teoría psicolingüística tiene en cuenta un sistema de señales o claves que el lector usa para leer y comprender el texto. Al respecto, Tracey y Morrow (2012) afirman que ésta enfatiza la idea de lectores como activos participantes dentro del proceso de lectura quienes construyen una explicación coherente y significativa del texto leído. Por su parte, en la teoría del lenguaje como un todo se precisa la lectura como un proceso natural que sólo se adquiere si los aprendices están inmersos en ambientes donde se exponen los aprendices a experiencias auténticas y significativas de lectura y alfabetización (Barrantes, Beltrán y Pérez, 2016).

Por otra parte, González, (2009) ve la metacognición como la capacidad del ser humano para conocer su propio modo de conocer, controlar su actividad cognitiva y los procesos que intervienen en ella, revisarlos y modificarlos en función de la nueva experiencia de aprendizaje. Finalmente, la teoría del enganche; la cual orienta a los educadores en ayudar a sus aprendices a estar más comprometidos con su propio aprendizaje y se percibe un lector comprometido, como aquel que está intrínsecamente motivado a la lectura (de la Calle et al., 2014). Son aprendices mentalmente activos, que utilizan sus estrategias metacognitivas para construir su propio aprendizaje. Además de incluir los elementos centrales de la teoría metacognitiva, tiene en cuenta los aspectos sociales, conceptuales y motivacionales del aprendizaje. (Tracey \& Morrow,2006)

\section{El proceso lector y el vocabulario}

Para Aebersold and Field (1997) un proceso lector no sólo comprende la relación entre el lector y el texto, también tiene en cuenta la influencia 
que ejercen algunos factores como la familia, la comunidad, la escuela, el ambiente sociocultural y las diferencias individuales. "Leer es un proceso dinámico que se desarrolla poco a poco, con diferentes actividades que ocurren en diferentes momentos" (p. 6).Por su parte, Aebersold y Field (1997) consideran la lectura como el proceso en el cual las personas observan un texto y le asignan significados a los símbolos que se encuentran escritos en ese texto. Por lo tanto, el texto y el lector son los elementos que se requieren para iniciar un proceso lector, la interacción entre ellos es lo que realmente constituye el proceso de comprensión de lectura.

Uno de los aspectos más importantes en el proceso de comprensión de lectura es precisamente obtener significado dentro de un texto; llegar a comprender la finalidad de este. Por lo cual la adquisición de vocabulario es fundamental para los aprendices de una lengua y así poder comprender el significado de las oraciones que conforman el texto.

Una de las premisas más comunes en aulas de clase donde se enseñe inglés como lengua extranjera, es precisamente, el no poseer el suficiente vocabulario para poder comprender el significado de un texto, razón por la cual se planteó este estudio, y se espera contribuir al proceso de aprendizaje de los futuros profesionales de este país.

\section{Metodología}

El propósito de este estudio fenomenológico es entender cuál es el papel que desempeña la enseñanza de vocabulario en el proceso de comprensión de lectura, desde la creencia de algunos docentes universitarios que desarrollan su labor pedagógica con estudiantes de pregrado que deben cursar la asignatura de inglés, como parte de su formación profesional y/o como requisito para obtener su título profesional en una universidad privada de la ciudad de Bucaramanga.

Según los aportes de Hernández, Fernández y Baptista. (2010) las investigaciones cualitativas comprenden un proceso lógico-inductivo, que va de lo particular a lo general. Es así, que se condujo este estudio, iniciando desde procesos particulares como entrevistas a los participantes, posteriormente análisis de datos obtenidos, revisión de resultados y documentos denominados guías cátedra, que hacen parte del insumo del 
instituto de lenguas para la ejecución de la labor docente y construcción de conclusiones.

Para el desarrollo de este proceso investigativo se consideraron las fases propuestas por Rodríguez, Gil y García (1999), las cuales corresponden a la forma como se investigó este fenómeno. En primer lugar, una fase preparatoria que incluye una etapa de reflexión personal de la labor pedagógica y se inicio con la búsqueda del estado del arte y/o teorías inmersas. Luego, se prosigue con la fase de trabajo de campo, en la cual se realiza la construcción de las preguntas, creación del formato de entrevista, aplicación del instrumento elegido y solicitud de documento institucional denominado guía cátedra. Posteriormente, la fase analítica la cual se lleva a cabo mediante el método de comparación constante de Glaser y Strauss (2008) el cual establece la creación de unos códigos comunes en los datos obtenidos y un análisis detallado de los mismos. Finalmente, una fase informativa en la cual se hace la presentación de los resultados y se generan las conclusiones de la presente investigación.

La población objeto de estudio corresponde a 4 docentes de inglés como lengua extranjera que laboran en una universidad de carácter privado de la ciudad de Bucaramanga, y que además tienen como mínimo 5 años de experiencia profesional. Como instrumento de recolección de datos se realizó una entrevista semiestructurada, ya que el presente estudio tiene como fin identificar, describir y entender cabalmente el fenómeno desde la perspectiva de sus actores. Así mismo, se recolectaron las guías cátedra de inglés, con las que cuenta el instituto de Lenguas para hacer un análisis descriptivo de ellas, y mirar hasta qué punto se observan incluidos los lineamientos requeridos para la adquisición de vocabulario en lengua extranjera.

Las entrevistas de los participantes fueron grabadas en audio, y luego transcritas. Se revisaron cuidadosamente las grabaciones y se realizaron las respectivas transcripciones varias veces, con el fin de obtener una transcripción bien detallada y confiable, y de esta manera, asegurar un entendimiento profundo de la información obtenida. Primero, se escribieron todos los datos generales, manualmente, de cada una de las opiniones de los sujetos participantes. Luego, esos datos generales se clasificaron por preguntas y se consignaron las respuestas de cada uno de los participantes según cada una de las preguntas de la investigación. Esta clasificación de la información se realizó con el fin de hacer una lectura minuciosa de la información línea por línea. Posteriormente, se 
identificaron las partes de la información relacionadas con la pregunta de investigación y se procedió con la redacción de notas de campo, que se realizaron de forma manual y que contenían comentarios y reflexiones acerca de los datos obtenidos.

Luego, se inició el proceso de codificación de la información, teniendo en cuenta la información relevante para dar respuesta a la pregunta de investigación. Producto de esta codificación se obtuvieron 53 códigos que se transcribieron nuevamente, pero esta vez en un documento de Word, previa revisión final de las grabaciones- insumo de la investigación. De acuerdo con McMillan y Schumacher (2005), un código en investigación es un nombre o una frase que se utiliza para proveer significado a la información que nos suministran los participantes. "Códigos pueden ser actividades, citas, relaciones, contextos, perspectivas de los participantes, eventos, procesos, o cualesquier otra acción o idea" (p.371).

Luego de obtener todas las codificaciones, se hizo una lista comparativa para revisar cuál de ellas era repetitiva. De esta manera, se logró agrupar las codificaciones y formar las categorías. "Categorías son entidades compuestas por grupos de códigos, las cuales se utilizan para dar significado a los códigos" (McMillan \& Schumacher, 2010, p.376).

Así, se clasificaron las categorías de acuerdo con su importancia, se les dio un nombre, y se escribió una definición para cada una de ellas. Finalmente, se vincularon las categorías emergentes del proceso de recolección de información y se creó un patrón con el cuál se logró entender un poco más las creencias y prácticas de los profesores acerca del fenómeno estudiado, no sólo a partir del análisis realizado a las entrevistas, sino también al análisis descriptivo de la guía cátedra.

\section{Resultados}

Luego del proceso de organización y preparación de la información para el análisis de los datos y de una reflexión profunda a partir de las intervenciones de los docentes, se obtienen 53 códigos en el proceso de codificación de la información. Estos se agruparon en tres categorías principales a saber: 
1. Vocabulario como herramienta de comunicación: Abarca los vocablos, expresiones y conjunto de palabras que se utilizan con fines comunicativos, mediante los cuales dos o más personas pueden llegar a acuerdos, establecer relaciones, etc.

2. Rol docente en la enseñanza del vocabulario: Papel o función que ejerce el maestro en cuanto a la enseñanza de léxico. Se incluye las estrategias que utiliza para hacer del proceso algo efectivo y significativo para sus aprendices.

3. Lectura y Vocabulario: Se pretende establecer la relación entre la enseñanza de vocabulario a través de diversos ejercicios derivados de la lectura.

\section{- ¿Cómo se llegó a la premisa Vocabulario como herramienta de comunicación?}

Los participantes de este estudio coinciden en la percepción del vocabulario como una herramienta de comunicación ya que es indispensable para la expresión de ideas, pensamientos, sentimientos, para llegar acuerdos, para establecer relaciones con otras personas y sólo esta enseñanza llega a ser efectiva cuando se logra que los estudiantes se comuniquen. En este momento, empiezan a tejerse las subcategorías expresión, contexto, aprendizaje y competencia, cada una de las cuales desempeña una función esencial en el desarrollo de la otra.

El estudiante necesita poder expresar sus ideas, sentimientos, y demás libremente, pero para hacerlo adecuadamente debe saber ubicarse dentro de un contexto, saber elegir las palabras adecuadas que necesita para tener una comunicación efectiva (Pérez, 2013). En este punto de la comunicación efectiva, debe conocer sus estilos de aprendizaje, la forma como su condición de aprendiz funciona para lograr desarrollar competencias, y por ende llegar a ser un ciudadano competente en cualquier ámbito personal o profesional, pero en una lengua extranjera, que, en este caso, es el inglés.

\section{- ¿Cómo se percibe el rol que desempeña el docente en la enseñanza de vocabulario?}

Los docentes tienen claro que, aunque el proceso de aprendizaje de lenguas es un trabajo que requiere toda la disposición y disciplina del aprendiz, es una responsabilidad compartida, y es el docente quien tiene 
en sus manos el conocimiento de teorías, y estrategias que se pueden aplicar para hacer que un aprendizaje en lengua meta sea exitoso.

Es esencial que un maestro posea fundamentos lingüísticos y pedagógicos, conozca de teorías de aprendizaje de lenguas, maneje diversos métodos y estrategias, pero lo más importante es que tenga la habilidad para compartir ese conocimiento a sus aprendices, que logre que los estudiantes se comuniquen dentro de un contexto real, que haga que realmente se de ese aprendizaje significativo, que esos nuevos vocablos lleguen a hacer parte de su lenguaje, y que lo use en su vida de forma natural.

Los docentes coinciden en afirmar que para lograr que ese aprendizaje de nuevos vocablos se lleve a cabo de forma significativa, es mejor realizar ejercicios que promuevan el uso de gráficos, ya sean mapas mentales, mapas conceptuales o redes semánticas, eso sí sin dejar de lado el contexto como eje fundamental de este proceso (Tahull, 2016). Aunque los docentes participantes refieren diversas estrategias que se pueden usar en el proceso de enseñanza de vocabulario, ninguno de ellos menciona que esas estrategias deben ser clasificadas de acuerdo con el nivel en el cual se esté dando la instrucción, porque depende de ello la cantidad de palabras y relaciones que los estudiantes puedan encontrar.

En este orden de ideas, es importante que el instituto de lenguas incluya en la guía cátedra la información pertinente a las estrategias que se pueden usar en las diferentes habilidades a desarrollar en el proceso de aprendizaje de inglés como lengua extranjera, y se otorgue un manejo más claro al aprendizaje del léxico.

\section{- ¿Cómo se percibe la relación entre lectura y vocabulario?}

Los docentes coinciden en afirmar que existe una conexión estrecha entre el proceso de lectura y el vocabulario, que una no se puede separar de la otra y que definitivamente todo radica en contextualizar la enseñanza. Si estamos contextualizando a los estudiantes, va a ser más fácil el proceso de comprensión, y por ende los estudiantes empezarán a adquirir nuevo léxico que luego hará parte de las expresiones o palabras que los aprendices usan normalmente. 


\section{Descripción de La Guía Cátedra}

La guía cátedra es el documento con el que cuenta el docente para desarrollar su labor, y ésta mantiene el mismo esquema para todos los niveles, es decir se incluye el nombre del curso, el código, los créditos, el número total de horas, las temáticas a desarrollar, la modalidad de estudio, entre otros.

Para iniciar el análisis del documento, se indagó dentro del mismo, información detallada sobre las competencias que se pretenden desarrollar en el curso correspondiente a esa guía, basadas en el Marco Común Europeo de Referencia para idiomas, que es la guía internacional usada por la universidad y reconocida por el gobierno nacional a través del ministerio de educación. Los estudiantes que cursan inglés en el instituto de lenguas de la universidad objeto de estudio necesitan demostrar sus conocimientos y habilidades al culminar sus cursos en los niveles A1, A2, B1, B2 respectivamente. Sin embargo, no se visualiza en la guía ninguna parte dedicada a describir cómo el componente de vocabulario de la lengua meta debe ser enseñado. Ninguna de las partes del formato contiene información relacionada a este respecto. Aunque se relacionan las temáticas que se desarrollaran en el nivel, no aparece descripción alguna sobre las palabras y/o expresiones idiomáticas que se introducirán en cada unidad a trabajar. Tampoco se describe ninguna actividad o técnica para trabajar vocabulario en ninguna de las habilidades, y por ende tampoco en lo concerniente a los diferentes momentos de la lectura; es decir, actividades antes, durante y después de la lectura.

Del mismo modo, tampoco se incluye dentro de la guía cátedra nigún criterio que se aplicará dentro de los momentos de evaluación, es decir en lo correspondiente a las notas de clase, los talleres de la plataforma, los exámenes mid-term y final exam, entre otros recursos evaluativos. El único parámetro que aparece estipulado para el proceso de evaluación es el porcentaje que vale cada actividad dentro del corte de notas. Es así como se observa que efectivamente este es el primer paso que debe darse, y consiste en realizar una modificación en el documento institucional.

\section{Conclusiones}

Mediante la presente investigación se logró resolver la pregunta planteada inicialmente: ¿Cuáles son las percepciones que tienen los 
docentes de inglés acerca del rol de la enseñanza de vocabulario en el proceso de comprensión de lectura de inglés como lengua extranjera en estudiantes de pregrado? Ya que las percepciones obtenidas del proceso de recolección de información fueron agrupadas en tres categorías a saber: Vocabulario como herramienta de comunicación, Rol docente en la enseñanza del vocabulario, y lectura y vocabulario.

El vocabulario es imprescindible para la expresión de ideas, para comunicarnos y establecer acuerdos, pero este debe enseñarse de forma contextualizada para que genere aprendizajes significativos en los estudiantes. No es suficiente reconocer que el vocabulario juega un papel importante en la enseñanza de una lengua extranjera y reducirla a la enseñanza de palabras mediante un listado de palabras nuevas. Se debe partir de una enseñanza contextualizada, hacer que los estudiantes usen el léxico nuevo de forma natural y que lo vinculen a las palabras que usan normalmente. Así, el estudiante adquiere una responsabilidad compartida en su proceso y modifica sus estilos de aprendizaje, poniendo en práctica nuevas estrategias que lo llevan a adquirir una mayor competencia en lengua meta. Al respecto, debe existir un cambio de perspectiva, si el léxico juega un rol principal en determinar la fluidez, el léxico debería recibir mayor atención en los procesos de enseñanza. Por lo tanto, los docentes deben sensibilizar este enfoque, incluyendo en sus prácticas pedagógicas algunos ajustes de tipo metodológico que permitan dar un espacio importante al léxico dentro de sus clases.

El docente debe estar en continuo crecimiento profesional y debe procurar mantenerse actualizado en estrategias y técnicas que propendan el mejoramiento del nivel de sus estudiantes. Está en sus manos dar a conocer esas nuevas posibilidades de generar conocimiento y hacer que sus aprendices cumplan con los requerimientos de las instituciones educativas en cuanto al nivel de competencia en lengua extranjera que debe tener acorde a los estándares internacionales.

Se requiere una modificación al documento guía cátedra ya que hay procesos que podrían mejorar si se cuenta con la información requerida y sobre todo que todos los docentes de ese nivel estén apuntando hacia la misma dirección, y así lograr aprendizajes significativos producto del trabajo en equipo. 


\section{Anexos}

Instrumento 1: Entrevista semiestructurada

Objetivo de la entrevista: Identificar la percepción de los docentes acerca del rol que tiene la enseñanza de vocabulario en el proceso de comprensión de lectura.

Participantes: Docente de Inglés.

Tiempo estimado: 45 minutos

Herramienta usada: Skype.

Recursos: Programa para grabar entrevista por Skype -- Copia de formato de consentimiento informado - formato de entrevista.

\section{Protocolo de la entrevista:}

- Saludo

- Contextualización de la entrevista

- Entrega de una copia de consentimiento informado

- Preguntas orientadoras

- Cierre

- Agradecimiento

- Despedida

Información general del docente:

Fecha:

Estudios realizados:

Edad:

Tiempo de experiencia docente:

\section{Preguntas}

1. ¿Cómo cree usted que la enseñanza de vocabulario puede ayudar a sus estudiantes a aprender inglés?

2. ¿Cuál cree usted que es el propósito de la enseñanza de vocabulario en el aprendizaje del inglés?

3. ¿Para usted qué significa enseñanza efectiva de vocabulario en el aprendizaje del inglés como lengua extranjera?

4. ¿Cuál cree usted que son los fundamentos teóricos más importantes que un profesor de inglés debe tener para la enseñanza de vocabulario?

5. ¿Cuáles son las estrategias que usted utiliza preferiblemente en sus clases para ayudar a sus estudiantes en el proceso de aprendizaje de vocabulario en inglés?

6. Dígame por favor las razones por las cuales usted utiliza ese tipo de actividades para la enseñanza de vocabulario en inglés

7. ¿Podría por favor dar ejemplos de la manera como usted enseña colocaciones o collocations en inglés? Colocaciones son combinaciones de 2 palabras que pueden y deben ir juntas, como, por ejemplo: In advance, to cause damage to, o growing awareness.

8. ¿Qué tipo de capacitación ha recibido sobre estrategias para la enseñanza de vocabulario en su área en el último año?

9. ¿Cómo cree usted que se puede conectar la enseñanza de vocabulario con la habilidad de comprensión de lectura?

10. ¿Existe algún otro aspecto en sus prácticas educativas con respecto a la enseñanza de vocabulario que usted le gustaría compartir conmigo? 


\section{Referencias bibliográficas}

Aebersold, J. A. \& Field, M. L. (1997) From reader to reading teacher: Issues and strategies for second language classrooms. Cambridge University Press.

Aparicio, O.Y. (2018). Las TIC como herramientas cognitivas. Revista Interamericana de Investigación, Educación y Pedagogía, RIIEP, 11(1). https://orcid.org/0000-0003-35356288

Aparicio, O.Y., \& Ostos, O.L. (2018). Las TIC como herramientas cognitivas para la investigación. Revista Interamericana de Investigación, Educación y Pedagogía, RIIEP, 11(1). https://orcid.org/0000-0003-3535-6288. https://orcid.org/0000-0002-6477-9872

Azevedo, S. R. J. (2014). La composición histórica de la educación religiosa como componente curricular. Revista Interamericana de Investigación, Educación y Pedagogía, RIIEP, 7(1). DOI: https://doi.org/10.15332/s1657-107X.2014.0001.03

Barrantes, H. A., Beltrán, J. E. P., \& Pérez, F. A. R. (2016). Perfil del estudiante de pregrado de la Facultad de Estudios a Distancia de la Universidad Militar Nueva Granada. Revista Interamericana de Investigación, Educación y Pedagogía, RIIEP, 9(2). DOI: https://doi.org/10.22490/25391887.1948

Colombia: Ministerio De Educación Nacional (MEN) (2013) Orientaciones para la implementación de proyectos de fortalecimiento de inglés en las entidades territoriales. Bogota D.C. Imprenta nacional.

Colombia: Ministerio De Educación Nacional (MEN). (2006) Formar en Lenguas extranjeras: Inglés iEl reto! . Bogotá. Imprenta Nacional.

Council of Europe. (n.d) Common European Framework of Reference for Languages: Learning, Teaching, Assessment. Tomado de http://www.coe.int/t/dg4/linguistic/ Cadre1_en.asp

Cresswell, W. J. (2014) Qualitative, Quantitative and Mixed Methods Approaches. Research Design. Los Angeles: Sage Publications.

de Almeida, R. R., Santos, M. F., \& Porto, J. C. (2016). Lectura de textos ficcionales y el enfoque escolar de literatura: contribuciones para una Pedagogía de la Elección. Revista Interamericana de Educación, Pedagogía y Estudios Culturales, 9(1), 35-51. DOI: https://doi.org/10.22490/25391887.1925

de la Calle, C. V., Malaver, M. O., Gallego, J. D. M., Rodríguez, M., Flórez, J. C., Henao, C. E. \& Saldaña, R. (2014). Aportes de los doctorados de educación en ciencia, tecnología y sociedad, desde la sistematización de sus investigaciones doctorales científicas y formativas, 2000-2010. Revista Interamericana de Investigación, Educación y Pedagogía, RIIEP, 7(1). DOI: https://doi.org/10.15332/s1657-107X.2014.0001.04 
Glaser, B. G. \& Strauss, A. L. (2008). The discovery of grounded theory: Strategies for qualitative research. New Brunswick: Aldine Transaction Publishers.

González, F. (mayo - agosto 2009). Metacognición y aprendizaje estratégico. Integra Educativa Vol. II / № 2, 127 -136. Tomado de http://www.revistasbolivianas.org.bo/ pdf/rieiii/v2n2/n02a05.pdf

Hernández, R., Fernández, C., Baptista M. (2010). Metodología de investigación. Edición 5. A Subsidiary of The mcgraw-Hill Companies, Inc. ISBN: 978-607-15-0291-9º

McMillan, J. H., \& Schumacher, S. (2005). Investigación educativa una introducción conceptual. Pearson educación.

Ministerio de Educación, cultura y deporte (2002). Marco Común Europeo de referencia para las lenguas: aprendizaje, enseñanza, evaluación. Tomado de http://cvc. cervantes.es/ensenanza/biblioteca_ele/marco/cvc_mer.pdf

Muñoz, M. E., \& Periáñez, M. J. A. (2013). Fundamentos del aprendizaje y del lenguaje. Barcelona, ES: Editorial UOC. Retrieved from http://www.ebrary.com

Pérez, T. H. P. (2013). Aproximaciones al estado de la cuestión de la investigación en educación y derechos humanos. Revista Interamericana de Investigación, Educación y Pedagogía, RIIEP, 6(1). DOI: https://doi.org/10.15332/s1657-107X.2013.0001.05

Rodríguez, G., Gil, J. Y García, E. (1999). Metodología de la investigación cualitativa. Málaga: Ediciones Aljibe

Rosenblatt, L. (1996). La teoría transaccional de la lectura y la escritura. Textos en contexto, 1, 13-71.

Tahull, J. (2016). Modernidad, educación y género. El proyecto inacabado. Revista Interamericana de Investigación, Educación y Pedagogía, RIIEP, 9(2), 159-178. DOI: https://doi.org/10.22490/25391887.1947

Tracey, D \& Morrow, L. (2012). Lenses on Reading. An introduction to theories and models. New York, NY. comprehension through explicit instruction in strategies. Rastros Rostros, 17 (31), 37 -52 Ediciones Universidad Cooperativa de Colombia. Impreso. Doi: http:// dx.doi.org/10.16925/ra.v17i31.1271

Viejo, C. M., Cabezas, I. L., \& Martínez, M. D. J. I. (2013). Las redes de académicas en la docencia universitaria. Revista Interamericana de Investigación, Educación y Pedagogía, RIIEP, 6(2). DOI: https://doi.org/10.15332/s1657-107X.2013.0002.03 